\title{
Combinatorial, site-specific requirement for heterochromatic silencing factors in the elimination of nucleosome-free regions
}

\author{
Jennifer F. Garcia, Phillip A. Dumesic, Paul D. Hartley, Hana El-Samad, and Hiten D. Madhani ${ }^{1}$ \\ Department of Biochemistry and Biophysics, University of California at San Francisco, San Francisco, California 94158, USA
}

\begin{abstract}
High-resolution nucleosome occupancy maps of heterochromatic regions of wild-type and silencing-defective mutants of the fission yeast Schizosaccharomyces pombe revealed that heterochromatin induces the elimination of nucleosome-free regions (NFRs). NFRs associated with transcription initiation sites as well as those not associated with promoters are affected. We dissected the roles of the histone H3K9 methyltransferase Clr4 and the HP1 proteins Swi6 and Chp2, as well as the two catalytic activities of the SHREC histone deacetylase (HDAC)/ ATPase effector complex. Strikingly, different DNA sites have distinct combinatorial requirements for these factors: Five classes of NFRs were identified that are eliminated by silencing factors through a mechanistic hierarchy governed by Clr4. The SHREC HDAC activity plays a major role in the elimination of class I-IV NFRs by antagonizing the action of RSC, a remodeling complex implicated in NFR formation. We propose that heterochromatin formation involves the deployment in several sequence-specific mechanisms to eliminate gaps between nucleosomes, thereby blocking access to the DNA.
\end{abstract}

[Keywords: Schizosaccharomyces pombe; heterochromatin; nucleosome-free regions; silencing]

Supplemental material is available at http://www.genesdev.org.

Received May 11, 2010; revised version accepted June 24, 2010.

Heterochromatin is a specialized type of chromatin in eukaryotes that is structurally and compositionally distinct from bulk chromatin. Functionally, it controls multiple fundamental aspects of chromosome biology, including kinetochore assembly, sister chromatin cohesion, recombination, and gene expression (Dillon 2004; Peng and Karpen 2008; Gartenberg 2009). Studies in a variety of systems have identified numerous chromatin-modifying enzymes as well as structural proteins that promote heterochromatin assembly and function. Perhaps the most well-known chromatin modification required for these processes is the methylation of Lys 9 of the N-terminal tail of histone H3 by the $\mathrm{Su}(\mathrm{var}) 3-9 / \mathrm{Clr} 4 / \mathrm{Kmt} 1$ family of histone methyltransferases, and its recognition by the chromodomains of HP1 family proteins (Lomberk et al. 2006; Grewal and Elgin 2007; White and Allshire 2008; Djupedal and Ekwall 2009).

Despite the importance of heterochromatin, our understanding of how this type of chromatin executes its functions is limited. For example, the mechanism of transcriptional silencing by heterochromatin is not un-

${ }^{1}$ Corresponding author.

E-MAIL hitenmadhani@gmail.com; FAX (415) 502-4315.

Article published online ahead of print. Article and publication date are online at http://www.genesdev.org/cgi/doi/10.1101/gad.1946410. derstood. Multiple distinct sequences can be subject to heritable repression when brought into heterochromatin in a conserved phenomenon called position effect. Given that heterochromatin was first defined cytologically as condensed chromatin (Heitz 1928), a reasonable proposal is that the histone methylases, HP1 family proteins, deacetylases, and chromatin remodeling enzymes required for silencing produce repression by forming condensed, inaccessible, or "closed" higher-order chromatin structures that impede the association of the transcriptional machinery with DNA (Dillon 2004). Indeed, experimental tethering of $\mathrm{HP} 1$ to a genomic site in mammalian cells results in cytological chromatin condensation (Verschure et al. 2005). Given that higher-order folding of nucleosomes in vitro is strongly influenced by the length of internucleosomal linker DNAs (Routh et al. 2008), such compaction would likely be facilitated by regular nucleosome spacing in vivo. Supporting this view, P-element transgenes integrated near pericentric heterochromatin in Drosophila display increased regularization of nucleosome spacing (Wallrath and Elgin 1995; Sun et al. 2001). Additional evidence that silencing may involve increased uniformity of nucleosome spacing has come from studies of the ACF chromatin remodeling complex in Drosophila. This complex, which shares its catalytic subunit with 
two other complexes-ISWI and NuRD-promotes even spacing of nucleosomes in vitro (Fyodorov et al. 2004). Flies lacking the ACF-specific subunit Acf1 display decreased periodicity of nucleosome spacing in bulk chromatin, as well as a defect in silencing of pericentric heterochromatin (Fyodorov et al. 2004). However, since the effect of Acf1 is global, whether the effect on bulk periodicity directly promotes silencing in heterochromatin is not clear.

Despite the appeal of the notion that higher-order folding of heterochromatin might render it generally inaccessible, several studies do not seem consistent with this concept. For example, P-element transgenes inserted into Drosophila heterochromatin that are associated with increased nucleosome regularity, as compared with euchromatic P-elements, do not show any difference in DNA accessibility, as assessed by micrococcal nuclease (MNase) digestion (Sun et al. 2001). Second, studies in the budding yeast Saccharomyces cerevisiae demonstrated that the inherent accessibilities of euchromatin and Sirdependent heterochromatin did not significantly differ from each other, but that binding of general transcription factors and RNA polymerase II (PolII) association is inhibited at heterochromatin (Sekinger and Gross 2001; Chen and Widom 2005). However, since S. cerevisiae lacks H3K9 methylation and HP1 proteins, whether the conclusion applies to systems that employ repressive histone methylation is not clear.

An important model system for the study of repressive histone methylation has been the fission yeast Schizosaccharomyces pombe. As in metazoans, heterochromatin formation requires the action of a histone methyltransferase, Clr4, which catalyzes the formation of the H3K9 methyl mark that serves as a binding substrate for three chromodomain proteins: Swi6, Chp1, and Chp2 (Thon and Verhein-Hansen 2000; Motamedi et al. 2008; Sadaie et al. 2008). Swi6 is an ortholog of metazoan HP1. Chp1 is a subunit of the RITS complex required for RNAi-dependent post-transcriptional silencing (Verdel et al. 2004). Chp2 is the second HP1 homolog of $S$. pombe and a subunit of the SHREC silencing effector complex (Motamedi et al. 2008), which promotes transcriptional silencing and inhibits the association of PolII with heterochromatin (Sugiyama et al. 2007). The SHREC complex is orthologous to the human NuRD complex, and contains two chromatin-modifying factors: a histone deacetylase (HDAC) subunit called Clr3, and an ATP-dependent chromatin remodeling enzyme subunit called Mit1 (Sugiyama et al. 2007). Both activities of SHREC have been shown to be involved in silencing, and a Southern blotting experiment showed that catalysisdefective mutants in either Mit1 or Clr3 altered the nuclease digestion pattern of a specific region of heterochromatin (Sugiyama et al. 2007). It was proposed that SHREC promotes a chromatin architecture that leads to higher-order compaction of heterochromatin responsible for transcriptional silencing (Sugiyama et al. 2007).

Several regions of the $S$. pombe genome are assembled into heterochromatin. These include the pericentric regions, subtelomeric sequences, the rDNA cluster, and the silent mating type cassette locus mat2/3 (Grewal and Jia 2007). The latter region contains two silenced loci-
mat2-P and mat3- $M$ - that donate genetic information to the mat1 locus during the process of mating type switching. At the mat2/3 locus, stable silencing requires specific sequences called REII, cenH, and REIII (Thon et al. 1999; Ayoub et al. 2000). cen $H$ is related to sequences found in the $d g$ and $d h$ pericentric repeats, and to $t$ th (telomericlinked helicase) genes found in subtelomeric regions (Hansen et al. 2006). Endogenous transcriptional units in the $c e n H$, the $d g$ and $d h$ repeats, and subtelomeric regions are subject to both transcriptional and RNAi-dependent post-transcriptional silencing (Volpe et al. 2003; Bühler and Moazed 2007; Sugiyama et al. 2007).

Given the well-characterized nature of heterochromatin in $S$. pombe, and the genetic tools available in this system, we sought to determine how its two HP1 family proteins (Swi6 and Chp2) and the three key chromatinmodifying enzymes required for silencing (Clr4, Clr3, and Mit1) collaborate to generate the primary structure of heterochromatin. To accomplish this, we employed a tiling microarray-based method for single-nucleosome position mapping developed in S. cerevisiae (Yuan et al. 2005) that uses MNase to generate mononucleosomalsized DNA fragments. This approach is feasible because $S$. pombe heterochromatin is generally accessible to MNase digestion (Takahashi et al. 2000; Jin et al. 2002; Smirnova and McFarlane 2002; Murakami et al. 2007). We report here the construction and analysis of high-resolution maps of nucleosome occupancies at endogenous heterochromatic domains in fission yeast, in both wild-type cells and mutants lacking individual silencing factors and/or activities. Our results uncover a new critical feature of heterochromatin, in which nucleosome-free regions (NFRs) are eliminated through the site-specific, combinatorial action of silencing factors.

\section{Results}

Nucleosome position and transcript maps of S. pombe heterochromatin domains

We designed two DNA microarrays containing 60-mer oligonucleotides representing both the Watson and Crick strands of the regions depicted in Figure 1A. One array includes three heterochromatic regions of the genome: cen 1, tel2R, and the mat2/3 silent mating type region (Fig. $1 \mathrm{~A}_{\text {; }}$ for design and uniqueness of probes, see the Materials and Methods; Supplemental Fig. S1). Also represented on this microarray were 30 euchromatic loci in regions flanking the heterochromatic regions described above, as well as six well-studied loci (mei4, act1, ade6, his3, nmt1, and gut2). As a further control, we designed a second microarray that represents a large, randomly chosen 160$\mathrm{kb}$ segment of euchromatin from chromosome I (indicated as the sod1-nup124 interval in Fig. 1A). Microarray features for each DNA strand were offset from their neighbors by 8 base pairs (bp), and there was a 4 bp offset between the two strands, giving a maximum resolution of 4 bp for nucleosome measurements.

Nucleosome occupancies were mapped by hybridizing probes derived from mononucleosomal-sized DNA obtained from $\mathrm{MNase}$ digestion of chromatin against probes derived 


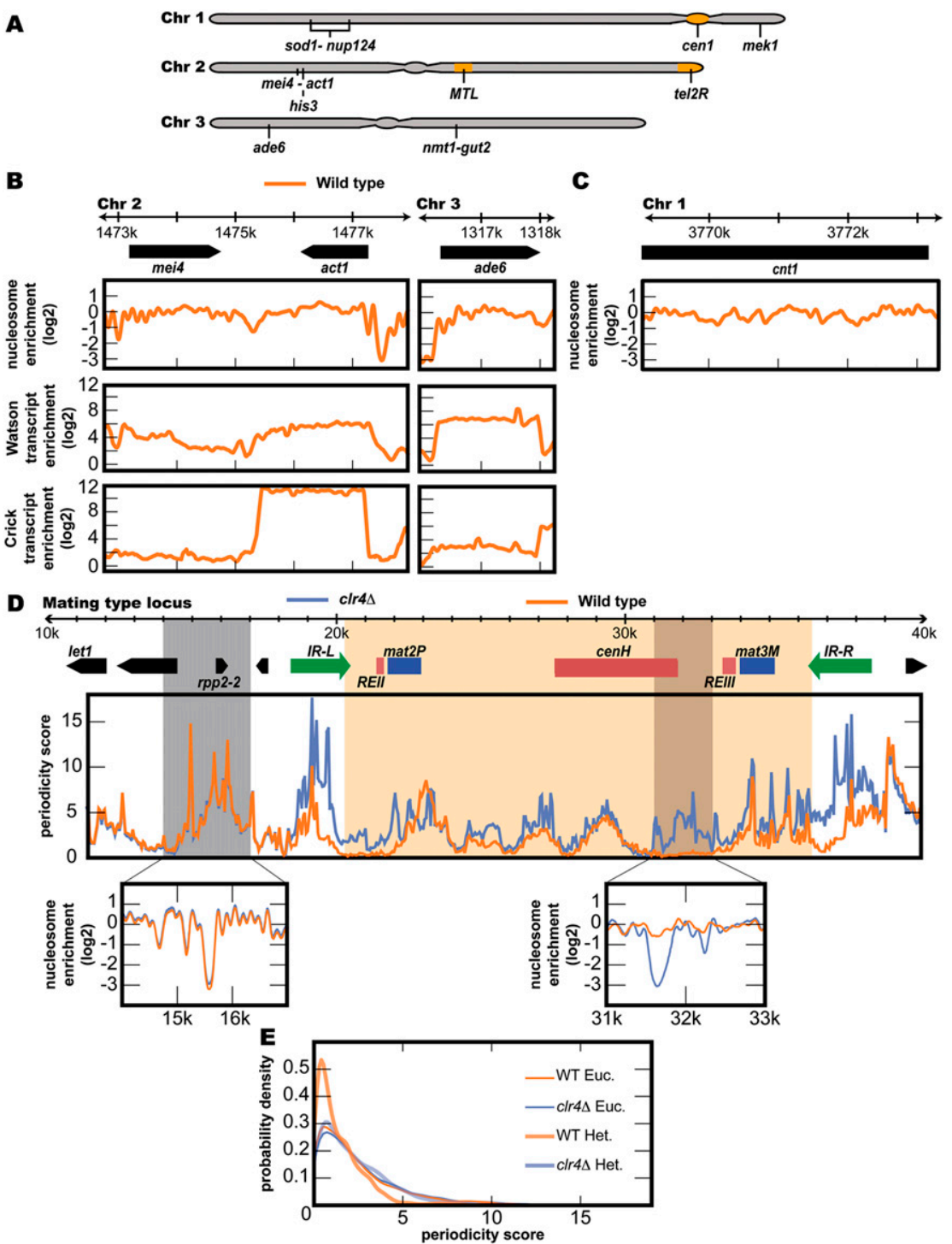

Figure 1. Analysis of nucleosome occupancy in $S$. pombe. (A) Array design. Schematic of the regions of the $S$. pombe genome covered by the two custom-designed tiling arrays as described in the text (note: not to scale). Orange boxes depicts heterochromatic regions present on the arrays. (B) Examples of control euchromatic genes. Moving average of normalized $\log _{2}$ nucleosome enrichment data over 19 probes (top data panels), and the moving average of $\log _{2}$-centered transcript enrichment data over 11 probes (two bottom data panels). $(C)$ Central centromere region chromatin structure. Data are shown as in $B$. (D) Periodicity analysis. Fourier analysis of the nucleosome occupancy data for clr4s (blue) and wild-type (orange) strains. The periodicity score for a sliding window of $1.2 \mathrm{~kb}$ (approximately eight nucleosomes) as a function of chromosome coordinate is plotted for the mating type locus. Nucleosome enrichment data for the regions highlighted in gray is shown in insets below the graph, and depict regions of high periodicity (left graph) and low periodicity (right graph, orange plot). (E) Normalized histograms of the periodicity scores. These were calculated for the euchromatin region between sod1-nup124 (thin lines) and the heterochromatin regions (thick lines) cen1, mat2/3, and tel2R. Histograms for wild-type (orange) and clr4s (blue) strains. from lightly digested genomic DNA. All experiments used a nonswitching (smt-O), $h^{-}$strain. Data described below are represented as the average of two biological replicates. The reproducibility of the data is summarized in Supplemental Figure S2.

Given that loss of silencing results in the accumulation of endogenous transcripts, we used the heterochromatin microarrays to map RNAs. These maps were generated by hybridizing probes derived from total RNA against probes derived from genomic DNA. Transcript maps were generated for both wild-type and clr4 $\Delta$ strains to identify Clr4-repressed transcripts. Since the nuclear exosome can degrade certain classes of PolII products, transcript maps were also produced in a strain lacking both Clr4 and the nuclear exosome component Rrp6 (clr4srrp64).

\section{Chromatin features of euchromatic loci}

We examined nucleosome occupancies at 36 euchromatic genes represented on the heterochromatin array and 53 euchromatic genes represented on the euchromatin array in order to validate our methods. Examples of three genes are shown in Figure 1B. The pattern observed at the adeb gene (Fig. 1B) was in good agreement with previous high-resolution studies of this gene (Song et al. 2008; Lantermann et al. 2009|. In agreement with studies in $S$. cerevisiae and other organisms, we found NFRs in the promoters of both active and repressed genes. In $S$. pombe, the meiosis-specific gene mek1 has been shown to display a small NFR in its repressed state (Wilhelm et al. 2008; Lantermann et al. 2009), and we recapitulated this observation using our methods (Supplemental Fig. S3D). We also observed a small NFR upstream of another meiosis-specific gene, mei4 (Fig. 1B). Additionally, a recent genome-wide study of euchromatic nucleosome positions describes a class of genes that maintain NFRs despite their repression by Tup1-related corepressors (Lantermann et al. 2010). Consistent with previous gelbased studies (Allshire et al. 1994), the central domain of cen1, which is not associated with H3K9 methylation, 
displayed more variable nucleosome positions (Fig. 1C). Additionally, the transcript map data delineated boundaries of expressed genes and intron removal events (Fig. 1B).

\section{Fourier analysis of nucleosome positions}

To assess whether there were silencing-specific changes in nucleosome spacing in the endogenous heterochromatin of $S$. pombe, we assessed the periodicity of nucleosome occupancy signal obtained from microarray hybridization by computing the Fourier transform of this signal. We defined a "periodicity score" for a window of data as the maximum peak in the Fourier power spectrum in the 120- to 240-bp frequency range, where a higher periodicity score indicates greater regularity of nucleosomal spacing. This range was chosen since it covers a large range of potential linker lengths. We then plotted this score for a 1.2-kb moving window as a function of the chromosomal coordinate (Fig. 1D; Supplemental Fig. S3A-C). As shown in the insets in Figure 1D, the periodicity score reflects what can be seen by eye as periodic versus nonperiodic patterns in the primary signal. To obtain a global view of these analyses, we plotted normalized histograms of the data for the sod1-nup124 randomly chosen euchromatic region versus the heterochromatic regions represented on the array for both wild-type and clr $4 \Delta$ samples. Surprisingly, the degree of periodicity in the heterochromatic regions was, on average, somewhat lower, not higher, than that of the euchromatic regions (Fig. 1E, cf. thick orange line and thin lines). Moreover, deletion of clr4 led to a modest increase in periodicity within heterochromatic regions (Fig. 1E). As might be expected, the distribution of periodicities of these regions in the clr $4 \Delta$ mutant becomes very similar to that of euchromatin (Fig. 1E, cf. thick blue line and thin lines).

\section{Clr4-dependent elimination of NFRs within heterochromatin}

To understand the decreased periodicity observed within heterochromatin, we systematically queried the data sets for DNA regions that displayed the largest differences in the nucleosome occupancy signals between the wild-type strain and the clr $4 \Delta$ mutant. Using an arbitrary cutoff of a fourfold difference, we identified 15 regions that each displayed such a difference between the wild-type and clr4 $\Delta$ signals (Fig. 2A-C; Supplemental Figs. S4-7, regions marked with gray boxes; Supplemental Table S4). Notably, each of these changes represented the appearance of an NFR in cells lacking Clr4. Many of these changes (nine of the 15) are associated with $d g$ and $d h$ centromeric repeats or sequences at other regions of the genome related to these repeats: We observed six at cen1, two at the cenH element of the mat2/3 locus, and one within the tlh2 gene near tel2R. Since $d g$-like and $d h$-like elements

\section{A}

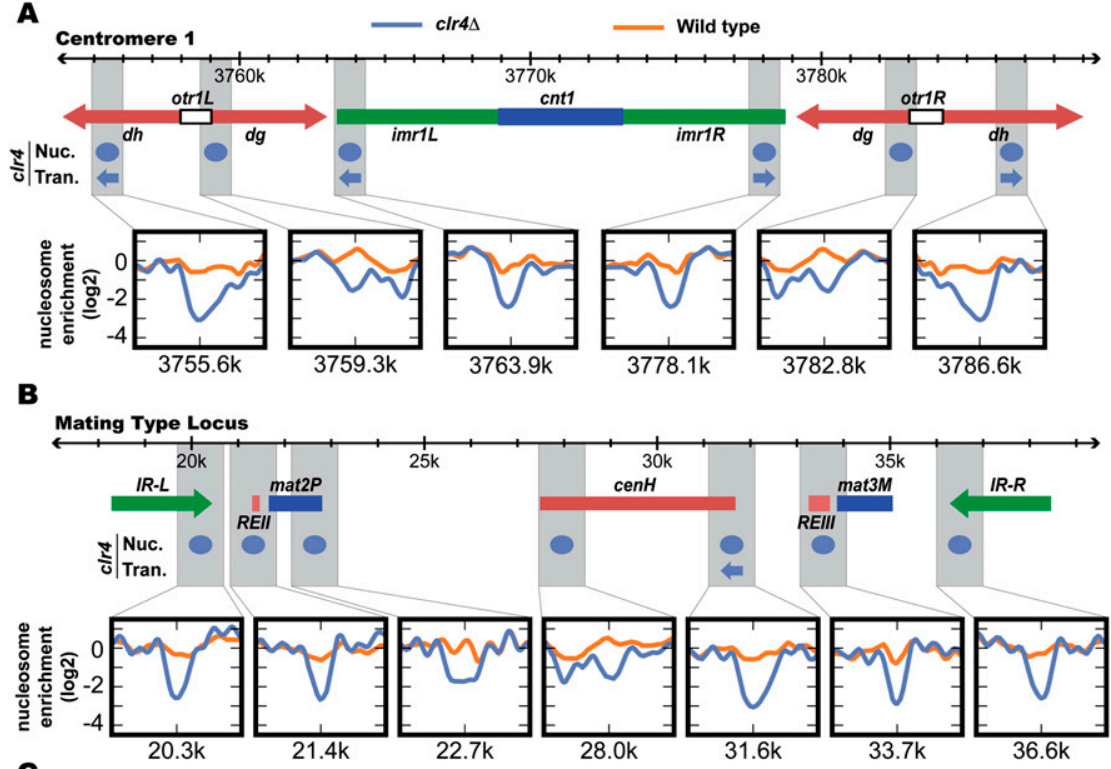

C

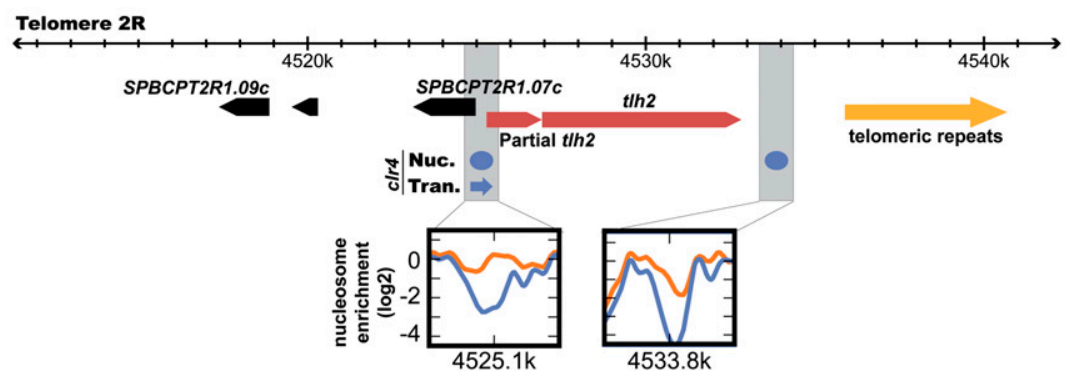

Figure 2. Clr4 promotes the elimination of NFRs. Annotated schematic for each heterochromatic region is displayed in $A-C$. The clr $4 \Delta$ and wild-type data sets were analyzed for regions that display a $\log _{2}$ difference of 2 or greater. All regions passing this cutoff are shown and highlighted by $1-\mathrm{kb}$ gray boxes centered on the maximum difference. Insets display the corresponding nucleosome enrichment data for wild- type (orange) and clr4s (blue) strains for that $1-\mathrm{kb}$ region. A blue circle within the gray boxes denotes each Clr4-dependent chromatin change. The blue arrows represent the appearance of a Crick (left pointing arrow) or Watson (right pointing arrow) Clr4-repressed transcript that initiates within the nucleosome-depleted region. Transcript mapping data are shown in Supplemental Figures S8-S10. The reproducibility of the replicate data is shown in Supplemental Figure S2. 
are known to contain Clr4-repressed transcription units, we compared our nucleosome occupancy maps with our transcript maps. We observed that six of the nine changes associated with $d g$ and $d h$ corresponded with transcript initiation sites in the clr4s strain but not in wild type (Fig. 2, gray boxes marked with blue arrows; Supplemental Figs. S8-S10). While the $d g$ and $d h$ repeats are not unique in the genome, there were sufficient unique probes in the cenH region of mat2/3 to demonstrate the observed effects occurred at $c e n H$, and not only at the related $d g$ and $d h$ repeats (Supplemental Fig. S1A).

Of the changes that were not within the $d g$ and $d h$ repeats or related elements (six of the 15), none corresponds to known promoters or detectable transcription initiation sites, even when the clr $4 \Delta$ rrp $6 \Delta$ double mutant was compared with wild type (Supplemental Figs. S8S10). Five of these changes occurred in the silent mat2/3 locus, while one was observed adjacent to the telomeric repeats (Fig. 2B,C). Interestingly, two of the NFRs that appeared were precisely at the REII and REIII regions of the mat $2 / 3$ domain that encode signals that promote heterochromatin formation (Fig. 2B). The eliminated NFR associated with REIII overlaps with DNA-binding sites for the silencing initiation heterodimer Atf1/Pcr1/Jia et al. 2004a). An NFR also appeared in the clr $4 \Delta$ mutant within the inverted repeats (IRs) that flank mat2/3; as with the REII and REIII NFRs, we observed no evidence for transcription initiation at these sites (Fig. 2B; Supplemental Fig. S9). Additionally, unlike the NFR associated with a centromeric $d g$ transcript, we do not observe a change in PolII occupancy at these sites in a clr $4 \Delta$ mutant when compared with wild type, further indicating that Clr4-repressed transcripts are not associated with these sites (Supplemental Fig. S11).

The silent mat2- $P$ cassette is unusual in that it contains two divergent transcription units (mat2-Pc and mat2-Pi) separated by a small $(\sim 120$-bp $)$ intergenic region. Within the mat2-P silent mating type cassette, an NFR appeared in the clr $4 \Delta$ mutant within the body of the mat-Pi gene, but not in the intergenic region (Fig. 2B). No transcript was detected in clr $4 \Delta$ cells from mat2-P, likely because activation of the mat2-Pc/Pi promoter occurs during nitrogen starvation and mating, whereas our experiments were performed in rich media in the absence of mating partners. We did not observe any changes to the silenced mat3-M locus, presumably because of the active copy of minus information present at the active mat1-M cassette. These data support a mechanism in which heterochromatin assembly generally suppresses the formation of NFRs, whether or not they are associated with transcription initiation sites.

In S. cerevisiae, nucleosomes that flank the NFRs are, as a class, the most well-localized nucleosomes (Yuan et al. 2005). It is therefore notable that the regions where periodicity of the nucleosome occupancy signal increases upon the loss of Clr4 are also regions where we observed the appearance of NFRs in the clr $4 \Delta$ mutant (cf. location of gray boxes in Fig. 2A-C and periodicity maps in Fig. 1D; Supplemental Fig. S3A,B). Thus, it is possible that the lower periodicity of silent heterochromatin is due to the lack of well-localized nucleosomes induced by NFRs. This is evident when the nucleosome occupancy for the clr $4 \Delta$ mutant is compared with that of wild type: Nucleosome peaks that flank the NFR generally appear to be more pronounced and periodic in the clr $4 \Delta$ strain than in the wild-type strain (see the low periodicity region in Figs. 1D [inset], 2).

\section{Silencing-resistant NFRs at heterochromatin boundaries and the SRE}

Two types of heterochromatin boundary elements have been characterized in $S$. pombe: tRNA genes in the innermost repeats (imrs) of the centromere (Scott et al. 2006), and regions within the IRs $I R-L$ and $I R-R$ that flank the mat2/3 heterochromatic domain. The activity for the latter requires a cluster of sites for the PolIII transcription factor TFIIIC (Noma et al. 2006). The mechanism of boundary activity is unknown, but has been suggested to involve tethering of boundary element chromatin to the nucleolus and nuclear envelope (Noma et al. 2006). Strikingly, at both the $I R$ and imr tRNA boundary elements, we observed NFRs whose structures remain unchanged in wild-type versus $c l r 4 \Delta$ strains (Fig. 3). Since H3K9
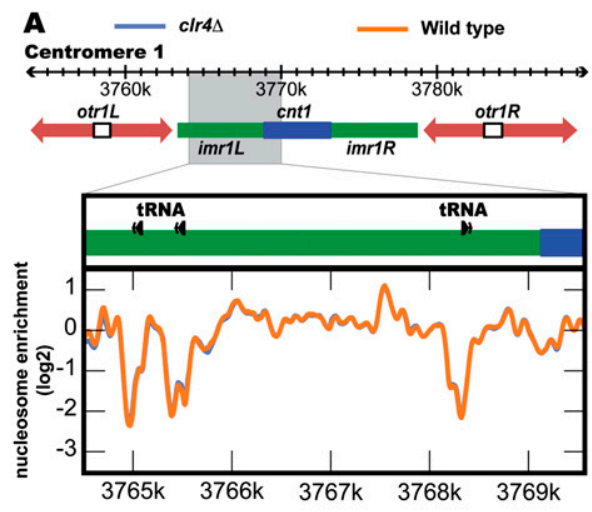

$\mathbf{B}$
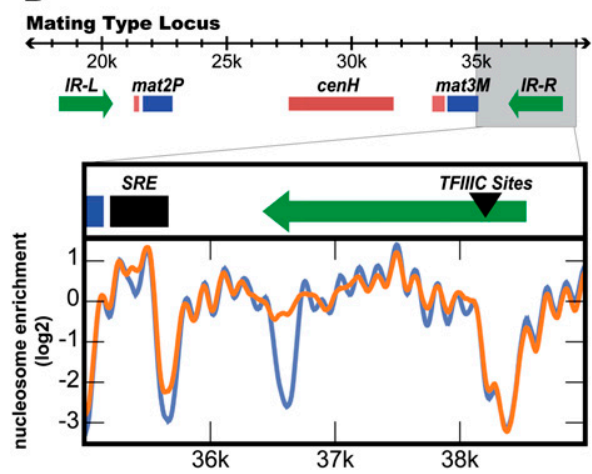

Figure 3. Silencing-resistant NFRs at tRNA and TFIIIC boundary elements. At the top of each panel is an annotated schematic of the either the centromere $1(A)$ or the silent mating type locus $(B)$. The region in the gray box depicts the region where the nucleosome enrichment for wild-type and clr $4 \Delta$ strains is shown. Additional sequence features found in the gray region are detailed above the nucleosome enrichment plot. SRE indicates the location of the Swi2-dependent recombination enhancer. 
methylation is known to spread from nucleation sites up to these regions, it appears that these NFRs are resistant to the NFR elimination mechanism.

Interestingly, an NFR that partially overlaps with a recombination enhancer (the SRE) involved in the directionality of mating type switching is also not affected by silencing (Fig. 3B). Since recombination itself requires heterochromatin formation, the accessibility of this region may be maintained by a specialized mechanism to ensure proper control of recombination. In addition, there appears to be a region within the recombination enhancer that displayed increased protection from digestion in wild type (Fig. 3B), consistent with previous studies indicating a role for this region in recruiting recombination factors in a manner dependent on heterochromatin (Jia et al. 2004b).

Distinct, site-specific requirements for HP1 family members in NFR elimination

We next extended our functional analysis to the two HP1 proteins of $S$. pombe that read the H3K9 methyl mark, Chp2 and Swi6. Because we expected that clr $4 \Delta$ mutants would have the strongest effect on nucleosome positioning of any heterochromatic factor, we used a more sensitive statistical method to conservatively determine the effects of other mutants on nucleosome occupancy at the 15 regions affected by Clr4 loss described in Figure 2 (see the Materials and Methods; Supplemental Fig. S12). Surprisingly, whereas Clr4 promotes the elimination of all 15 NFRs, this was not the case for the HP1 proteins. As indicated by the purple circles in Figure 4 and Supplemental Table S4, we observed significant differences between wild-type and chp2s cells at 11 of the 15 NFRs. In each of these cases, the nucleosome occupancy for chp2s was lower than the nucleosome occupancy for wild type and similar to that of the clr4s mutant (Fig. 4, cf. purple lines and blue and orange lines in plots). Notably, all six NFRs that were associated with transcription initiation in the $c l r 4 \Delta$ mutant require Chp2 for their elimination. In contrast, NFRs eliminated by Clr4 at the $d g$ repeat, within the mat2P loci, and at the left end of cenH did not require Chp2.

Strikingly, Swi6 controlled only NFR elimination at the mat2/3 locus (Fig. 4B, green dots; Supplemental Table S4). It did not affect nucleosome positioning at the
A

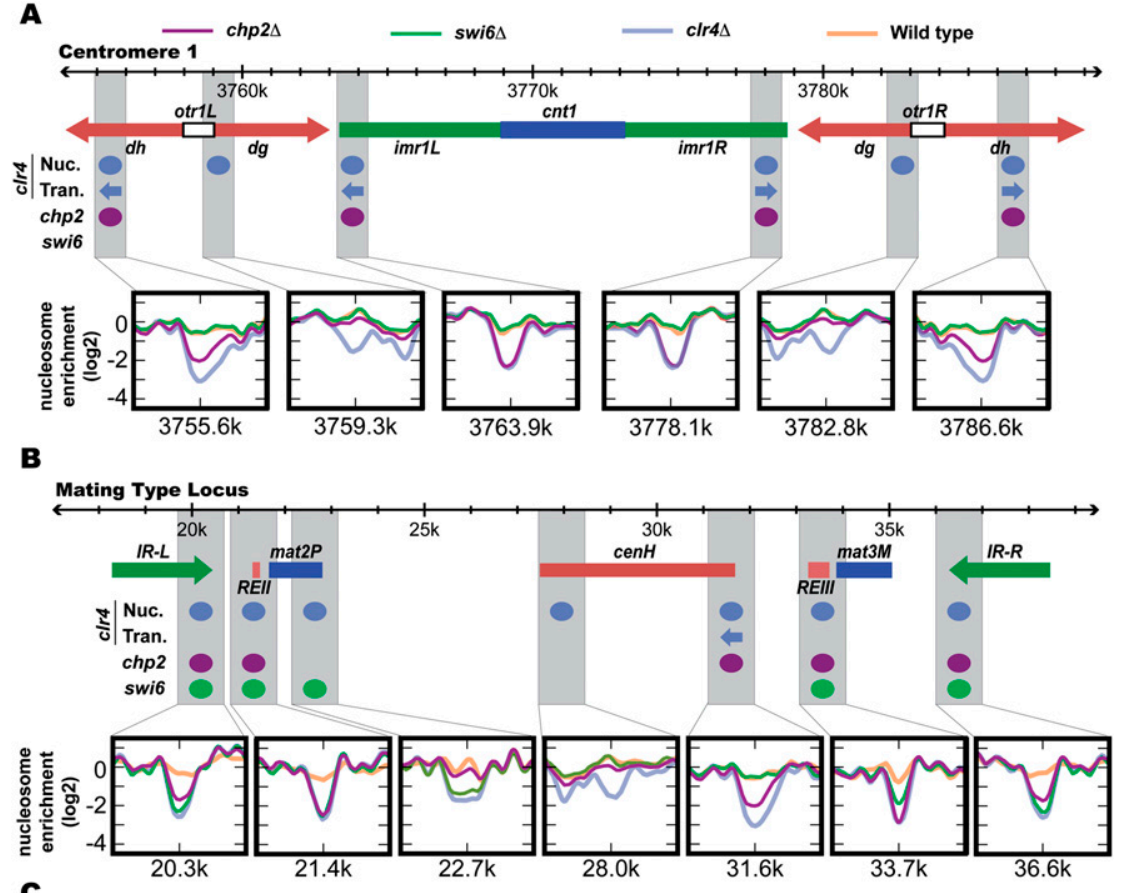

C

Telomere 2R

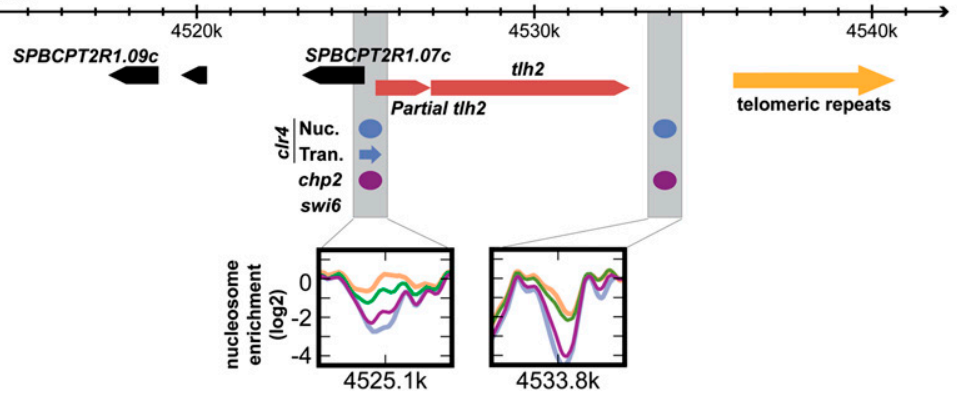

Figure 4. HP1 proteins Chp2 and Swi6 differentially affect NFR elimination. An annotated schematic for the indicated heterochromatic region is displayed at the top of each panel. The gray boxes depict $1-\mathrm{kb}$ regions that contain a fourfold greater change in nucleosome enrichment between clr4s and wild type as determined in Figure 2. Insets connected to and below the gray boxes contain plots of the nucleosome occupancy for chp2s (purple), swi6s (green), clr4s (blue). and wild-type (orange) strains within that $1-\mathrm{kb}$ region. Within the gray box, significant changes (see the Materials and Methods) between the mutant and the wild type are represented by colored circles (Chp2 dependence is represented with a purple circle, while Swi6 dependence is represented with a green circle). 
Garcia et al.

centromere or within the centromere-related cenH sequence at the mat2/3 locus (Fig. 4). Four of the five NFRs in mat2/3 that require Swi6 for their elimination also require Chp2 (Fig. 4). In contrast, the NFR associated with the mat $2 P$ locus requires Swi6 but not Chp2 for its elimination. Together, these data define four classes of NFRs eliminated by Clr4 action: those that require both HP1 proteins, those that require only one or the other, and those that do not require either HP1 protein.

\section{Dissection of the roles of silencing-associated enzyme activities in NFR elimination}

Previous analysis of catalytically defective mutants of the Clr3 deacetylase or the Mit1 ATPase subunits indicated that both deacetylase and ATPase activities of SHREC were important for silencing, and appeared to similarly change the nuclease digestion pattern of a region of chromatin upstream of mat3- $M$ when examined by gel-based methods (Sugiyama et al. 2007). Additionally, Chp2 has been purified as part of SHREC (Motamedi et al. 2008), raising the possibility that the function of Chp2 might be mediated by either the ATPase or HDAC activity of SHREC. We therefore examined whether NFRs appear in catalytically dead point mutants of Clr3 or Mit1. These alleles were used to replace the endogenous genes by homologous replacement (for details about construct, see the Supplemental Material; Supplemental Fig. S13). For 12 of the 15 sites examined, the HDAC-defective clr3D232N mutant displayed changes in the nucleosome occupancy profile that were similar to those observed in the clr4s mutant (Fig. 5, red dots; Supplemental Table S4). For 11 of the 12 of these regions, chp $2 \Delta$ had a phenotype similar to that of the catalytically dead clr3 allele. Thus, in these cases, the changes observed in clr4s and chp2s cells can be explained by a requirement for recruitment of the HDAC activity of SHREC by H3K9 methylation. However, in three cases, elimination of an NFR required Clr4 but neither Chp2 nor Clr3 activity (Fig. 5). Finally, in one case, elimination of the NFR associated with the
A

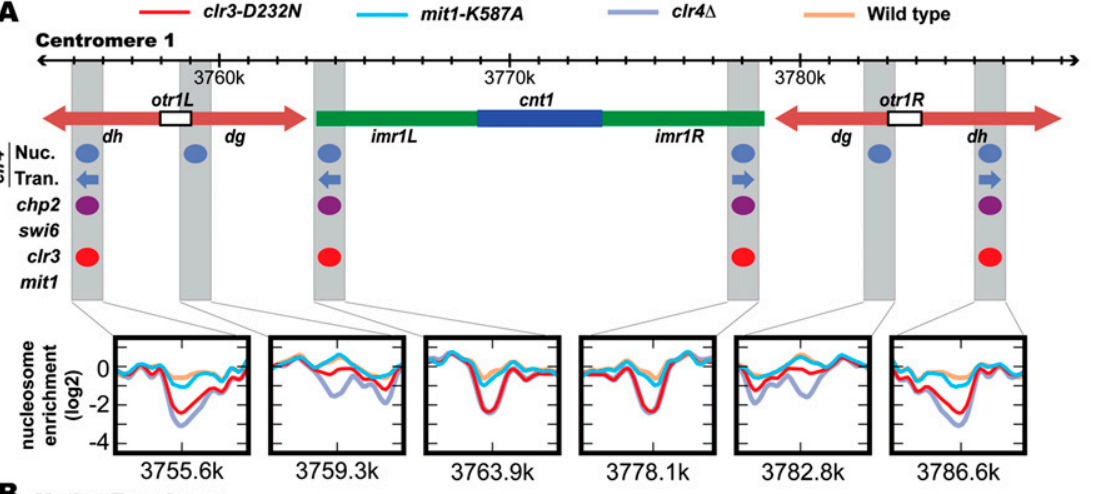

B Mating Type Locus

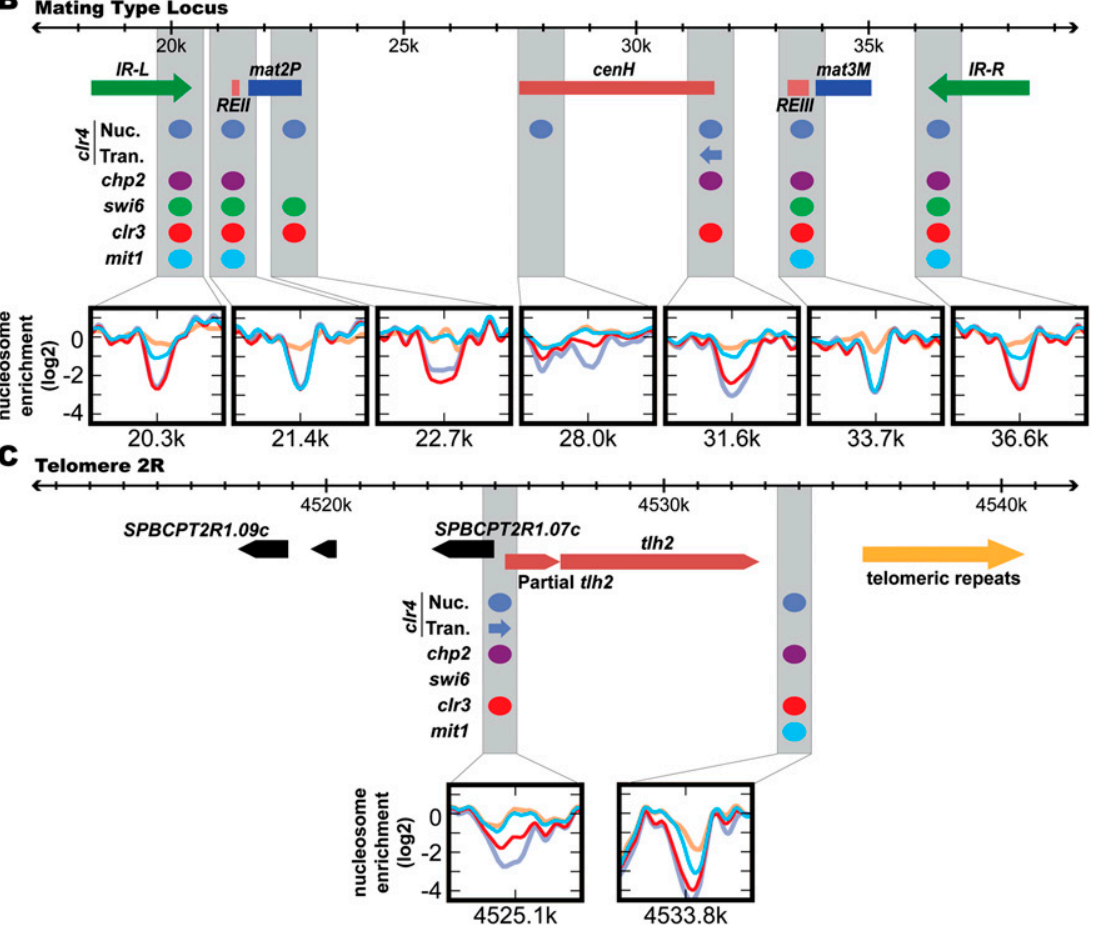

Figure 5. Silencing-specific changes at NFRs require specific activities of SHREC. Data for wild type (orange), the clr3-D232N mutant (red), the mit1-K587A mutant (bright blue), and the clr4 $\Delta$ mutant (blue) are plotted as in Figure 4. Significant changes between the wild type and the mutant are depicted by colored circles $/ \mathrm{Clr} 3$ dependence is represented by a red circle, while Mitl dependence is represented by a bright blue circle), and depict the genetic requirement for that factor in eliminating that NFR. 
mat2P was observed to require both Clr3 activity and Swi6 but not Chp2 (Fig. 5B). This result may explain previous observations that Swi6 appeared to be involved in SHREC recruitment at some but not all sites tested (Sugiyama et al. 2007).

The role of the ATPase activity of SHREC was distinct from that of its HDAC activity. In contrast to the clr3D232N mutant, the mit1-K587A mutant had no effect on the chromatin architecture of cen1. However, it did affect four sites in the mat2/3 locus and one site near tel2R (Fig. $5 \mathrm{~B}, \mathrm{C}$, bright blue dots; Supplemental Table S4). The most striking of these changes were associated with the REII and REIII silencing initiation signals in the mat2/3 region (Fig. $5 \mathrm{~B}$ ). This result is consistent with a previous gel-based assay that showed the appearance of an MNasehypersensitive site within the REIII region in a mit1K587A mutant (Sugiyama et al. 2007). Here, we found that the loss of the catalytic activity of Mit1 produced a phenotype very similar to that of the loss of Clr4 or either HP1 protein. These results imply an unexpected specialized role for the ATPase subunit of SHREC in remodeling NFRs associated with silencing initiation signals, but not transcription initiation signals. It is also worth noting that the NFRs in the inner portions of the IRs of mat2/3 also require Mit1, and have also been associated with weak silencing initiation signals in early studies (Ekwall et al. 1991).

In addition to its requirement for the recruitment of SHREC to other sites, Swi6 has been found to associate with a large number of chromatin proteins (Motamedi et al. 2008; Fischer et al. 2009), several of which have been shown to have relatively mild effects on silencing.
These were the two chomodomain-containing ATPdependent remodeling enzyme homologs Hrp1 and Hrp3, and the FACT complex subunit Pob3 (Jae Yoo et al. 2002; Walfridsson et al. 2005; Lejeune et al. 2007). However, with one exception, cells lacking any of these factors did not show statistically significant changes in heterochromatin structure (Supplemental Fig. S12; data not shown). The exception was curious: $h r p 1 \Delta$ cells display an increase in histone occupancy in the region adjacent to the telomere repeats of tel2R compared with wild type (Supplemental Fig. S14).

\section{Genetic requirements for NFR elimination define five classes of NFRs within heterochromatin}

These data suggest that the key players involved in controlling nucleosome positioning in heterochromatin are the histone H3K9 methyltransferase, the HP1 proteins, and the SHREC effector complex recruited by the HP1 proteins. Strikingly, their requirement is not uniform, but differs dramatically at different sites. As summarized in Figure 6, the 15 NFRs eliminated by Clr4 fall into five different classes (labeled class I-V in Fig. 6), based on the genetic requirements for their elimination in heterochromatin. In many cases, these dependencies correlate with known functional roles of the sequences involved /see the Discussion).

\section{A RSC mutant bypasses the requirement for Clr3 HDAC activity in NFR elimination}

To probe the mechanism by which silencing factors function to eliminate heterochromatic NFRs, we focused

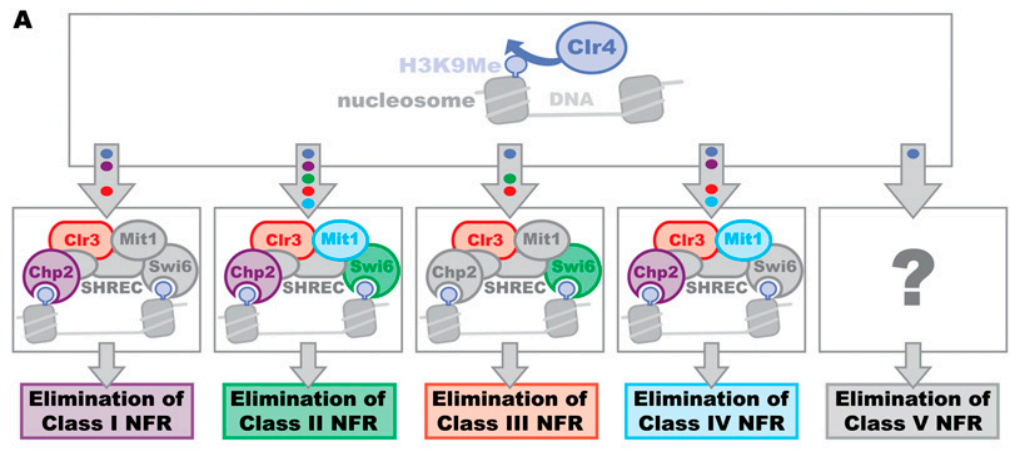

$\mathbf{B}$

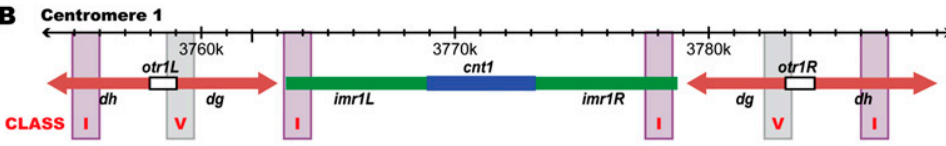

Mating Type Locus

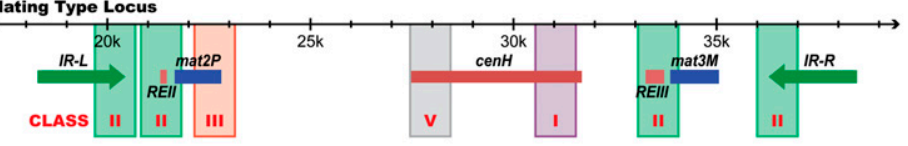

Telomere 2R

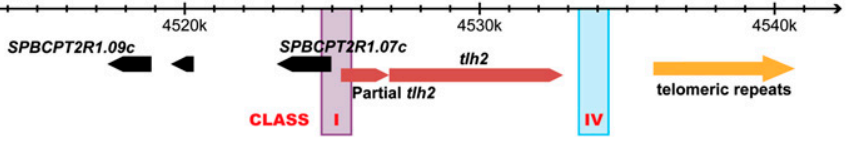

Figure 6. Combinatorial, site-specific requirements for NFR elimination. (A) Genetic dependences for the elimination of five different classes of NFRs within heterochromatin. For each class, the silencing factors required for the elimination activity are shown in color, while those that do not show a dependency are shown in gray. Colored circles in arrows summarize genetic dependencies identified in Figures 2, 4, and 5. (B) Location of NFR classes. Each change is color coded and labeled according to the classification shown in $A$. 
on how the Clr3 HDAC activity promotes NFR elimination. Previously, we uncovered a role for the RSC complex, an essential ATPase remodeling enzyme, in forming a subset of NFRs in S. cerevisiae (Hartley and Madhani 2009). Additionally, the RSC complex has the ability to recognize acetylated nucleosomes (Kasten et al. 2004; Carey et al. 2006; Hassan et al. 2007) through its multiple bromodomain-containing subunits. We hypothesized that the RSC complex and its role in forming NFRs is antagonized by the HDAC activity of Clr3 within heterochromatin. This hypothesis predicts that the necessity for Clr3 HDAC activity in NFR elimination should be bypassed by impairing the activity of RSC, particularly by inactivating its bromodomain-containing subunits.

While the $S$. pombe RSC complex is essential, it has three bromodomain-containing subunits-Rsc1, Rsc4, and Rsc58-for which viable knockouts can be generated (Monahan et al. 2008). Despite these findings, we were only successful in constructing a deletion of rsc1 in a wild-type strain and in a strain harboring the HDAC-dead point mutant $c$ lr3-D232N by transformation. We assayed these strains for nucleosome occupancy in heterchromatin and compared them with the wild-type and clr3D232N nucleosome occupancies (Fig. 7A-C; Supplemental Fig. S15A-C). We observed strong suppression of the clr3-D232N phenotype at four of the 12 NFRs by rsc1s (those associated with the $d h, c e n H$, and th2 elements) (Fig. 7A-C) and partial suppression at seven of the eight remaining NFRs. It is notable that the sites of strong suppression all correspond to class I NFRs; furthermore, all other members of class I show partial suppression by rsc1 1 . Importantly, the single $r s c 1 \Delta$ mutant did not affect heterochromatic nucleosome positions (Supplemental Fig. S15).

To mechanistically probe the suppression phenotype, we examined how clr3-D232N affected RSC recruitment to these regions. We used homologous recombination to epitope tag the essential catalytic subunit of RSC, Snf21, with a C-terminal CBP-2xFlag tag. We performed chromatin immunoprecipitation (ChIP) in wild-type and
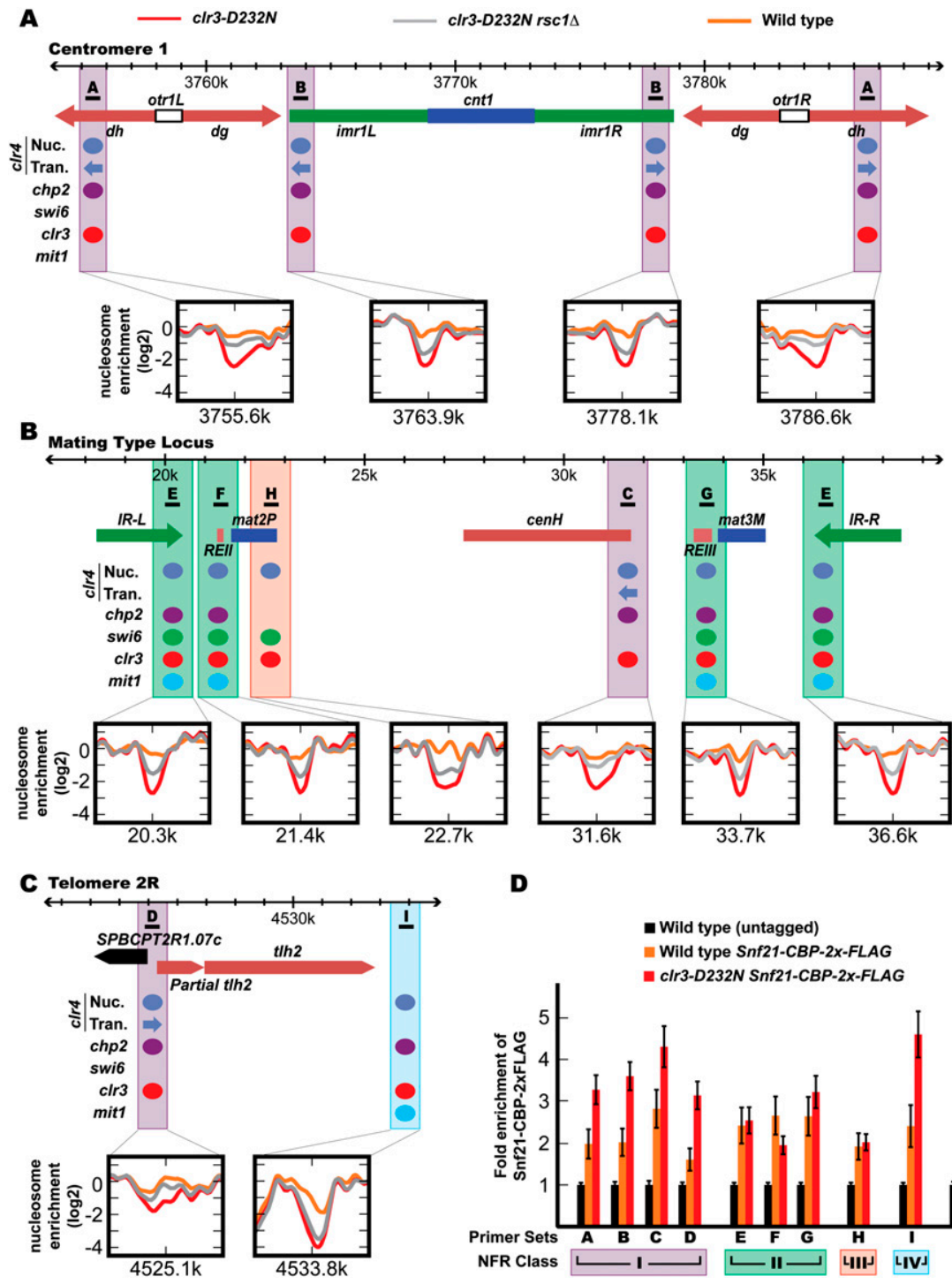

D

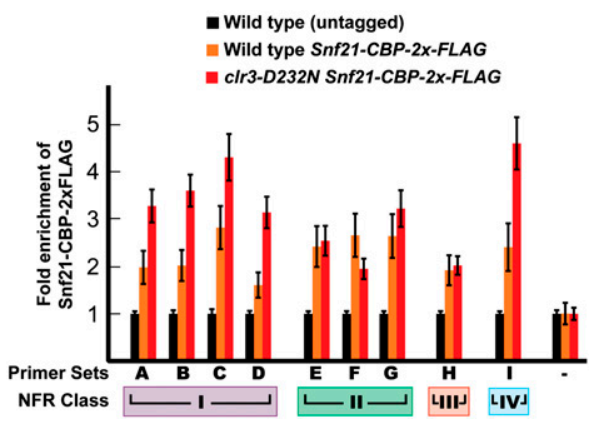

Figure 7. $r s c 1 \Delta$ mutation can suppress the requirement for Clr3 activity for NFR elimination. $(A-C)$ Plotted are the nucleosome enrichment data for clr3-D232N mutant (red), wild type (orange), and clr3-D232N rsc1s double mutant (gray) for each NFR. Data for rsc1s single mutant are shown in Supplemental Figure S15. Genetic dependences for NFR elimination are depicted by colored circles. $(D)$ Normalized ChIP data for Snf21-CBP-2XFlag in wild-type (orange bars) and clr3-D232N mutant (red bars) strains. The untagged control is shown in black. The locations of the quantitative PCR primer sets used are depicted in $A-C$, just below the chromosome coordinates. Primer sets are labeled according to the corresponding NFR class defined in Figure 6 . The negative control primer set $(-)$ is directed to mitochondrial DNA. Error bars represent the standard error for four independent immunoprecipitations. For normalization, the enrichments were first determined by calculating the immunoprecipitate to input ratio for each primer set, with the enrichment observed in the wild-type untagged strain set to a value of 1 . For each genotype, these enrichment values were divided by the respective enrichment value, determined using a negative control primer set directed against mitochrondrial DNA. 
clr3-D232N strains to analyze whether RSC recruitment to heterochromatin is affected by the HDAC activity of clr3. At regions for which rsc1s had the largest suppression effect on the clr3-D232N phenotype (namely, the class I NFRs), we observed a modest but reproducible increase in Snf21 occupancy in the clr3-D232N strain as compared with the wild-type strain (Fig. 7D, primer sets A-D). Together, the suppression results and the increased RSC recruitment in the clr3-D232N mutant strongly suggest that a key role of the Clr3 HDAC activity in NFR elimination is to limit recruitment of RSC. However, other classes of NFRs, including classes II and III, showed similar levels of RSC occupancy in wild-type and clr3D232N strains (Fig. 7D, primer sets E-H), suggesting that other mechanisms are at play (see the Discussion). The strongest reduction in RSC recruitment between the wild type and clr3-D232N mutant corresponded to the single class IV NFR adjacent to the telomeric repeats, at which the rsc1s mutation did not suppress the clr3-D232N phenotype (Fig. 7C). Thus, it appears that limiting access of RSC to this particular site is not sufficient to bypass the requirement for Clr3 activity, and further supports the notion that NFR elimination at distinct sites occurs via distinct mechanisms. This one example notwithstanding, these data point to a major role of the HDAC activity of Clr3 in promoting heterochromatin structure by antagonizing the NFR-promoting activity of RSC.

\section{Discussion}

\section{Heterochromatin induces elimination of NFRs}

To understand the mechanism by which heterochromatin assembly results in repression, we constructed nucleosome occupancy maps of fission yeast heterochromatic domains. We linked our observations to functional requirements by comparing profiles of wild type and mutants defective in one of three different chromatin-modifying enzymes required for transcriptional silencing: Clr4 histone methyltransferase, the Clr3 HDAC, and the Mit1 ATP-dependent chromatin remodeling enzyme homolog. As the latter two are subunits of the SHREC effector complex, we constructed catalytically defective point mutants to analyze their respective functions. We also examined the roles of the two $S$. pombe HP1 orthologs, Chp2 and Swi6. Fourier analysis revealed that periodicity of nucleosome positions was lower in heterochromatin than in euchromatin. Moreover, loss of Clr4 resulted in increased periodicity within heterochromatin. Our data show that $S$. pombe heterochromatin, unlike Drosophila heterochromatin, does not exhibit broadly increased regularity of nucleosome positions. Moreover, we found that five of the silencing factors we tested acted at specific sites to promote the elimination of NFRs. A simple model consistent with our data is that silencing factors induce NFR elimination to promote silencing by occluding binding sites of factors required for transcription initiation. Supporting this model is a previous observation that Chp2 and Clr3 but not Swi6 limit the association of PolII with the $d g$ and $d h$ repeats (Motamedi et al. 2008), sites that we found to require Clr4, Chp2, and
Clr3 but not Swi6 for NFR elimination. Such a model offers a simple physical explanation for transcriptional repression that does not require invoking a closed higherorder structure. Additionally, our results are also consistent with anecdotal reports of promoter-localized changes in silenced regions in S. cerevisiae (Vega-Palas et al. 1998), Drosophila (Sun et al. 2001), and humans (Chen and Yang 2001). Given that nucleosomes flanking NFRs are more periodic than those elsewhere in the genome (Yuan et al. 2005), NFRs may act to promote periodicity of nucleosomes in euchromatin, and thus the lower periodicity observed in $S$. pombe heterochromatin could be a consequence of NFR elimination.

\section{Cause versus effect}

An important issue historically has been whether changes in nucleosome organization associated with changes in transcription are a cause or an effect (Wu et al. 1979). Identification of mutations in chromatin-modifying enzymes and in histones themselves that affect transcription provided strong evidence that chromatin changes can cause changes in transcriptional behavior (Kayne et al. 1988; Brownell et al. 1996). Likewise, the fact that chromatin-modifying enzymes examined in our studies have been shown previously to be required for silencing in $S$. pombe heterochromatin argues that changes in chromatin structure of some type are a cause rather than consequence of transcriptional repression. Two lines of evidence suggest that NFR loss in heterochromatin induced by silencing factors corresponds to such a change. First, many of the eliminated NFRs that were observed did not colocalize with detectable sites of transcription initiation in the clr $4 \Delta$ mutant background, and, indeed, in several cases corresponded to silencing initiation elements. NFRs found at the REII and REIII initiation elements in clr4D cells may reflect nucleosome exclusionary mechanisms that allow the initial access of DNA-binding factors to these sites to initiate the silencing process, which is then reversed once silencing is established. Second, the transcriptionally inactive genes in euchromatin we examined display NFRs, indicating that loss of transcription per se is insufficient to produce a loss of NFRs. These observations are consistent with studies in $S$. cerevisiae indicating that NFRs are generally maintained at repressed genes (Yuan et al. 2005), and very recent studies in $S$. pombe showing that a major class of repressed genes, those targeted by Tup1-related corepressors, maintain large NFRs (Lantermann et al. 2010). Together, these data indicate that NFR elimination observed in heterochromatin cannot be explained by proposing that it is a consequence of shutting down transcription, but rather is an active process facilitated by chromatin-modifying activities.

\section{Distinct silencing factors are required at distinct sites to eliminate NFRs}

In addition to challenging the notion that reduced DNA accessibility is solely a consequence of a closed higherorder structure, our genetic data lead to another unexpected conclusion: Different activities of the silencing 
apparatus are required at different sites to enable similar structural changes; namely, the elimination of NFRs. The broadest effects were observed for Clr4, the HDAC activity of Clr3, and the HP1 homolog Chp2. The ATPase activity of Mit1 and the other HP1 homolog, Swi6, each had progressively more region-specific functions. Our analysis defined five classes of NFRs that had distinguishable, combinatorial genetic requirements for their elimination. As discussed further below, correlations were observed with the function of the DNA site involved. While additional work will be required to understand the mechanistic basis for these differential requirements, the results strongly imply that silencing factors perform different tasks at different DNA sequences. Because the placement of unrelated gene sequences into heterochromatin results in their silencing, this phenomenon has been called position effect. Implicit in this term is the concept that a sequence-independent mechanism, such as the formation of a closed higher-order structure, leads to silencing. An alternative possibility suggested by our studies is that the apparent sequence independence of position effect is a result of the ability of the silencing machinery to recognize and eliminate a general feature of euchromatin - the NFR - that is specified by the underlying DNA sequence. In this scenario, the site-specific requirements for combinations of silencing factors reflects the fact that different NFRs are programmed by different mechanisms (Hartley and Madhani 2009; Segal and Widom 2009a,b).

\section{Correlations between sequence functions and genetic requirements}

We observed several intriguing correlations between the genetic requirements for the elimination of particular classes of NFRs and the known functional roles of the underlying sequences. Class I NFRs, whose elimination requires both Chp2 and Clr3 activity, coincide with initiation sites for Clr4-repressed transcripts and sites of RNAi-dependent silencing (Fig. 6B). It is possible that Chp2 and Clr3 activity is particularly relevant to repressing transcription from these regions after the S-phasespecific accumulation and processing of transcripts by the RNAi machinery (Chen et al. 2008). Elimination of class II NFRs required Clr4, both HP1 proteins, and both activities of SHREC (Fig. 6A). These were restricted to the tightly repressed mat2/3 locus, and included the wellcharacterized silencing initiation elements REII and REIII (Fig. 6B). As mentioned above, NFRs eliminated in the inner portions of the IRs also fell into this class, and have been associated with silencing signals as well (Ekwall et al. 1991). The sole class III NFR is associated with the mat2P cassette (Fig. $6 \mathrm{~B}$ ), and its elimination requires the activity of Clr3 and Swi6 but not Chp2, indicating that Clr3 can act independently of Chp2, even though both are components of SHREC (Motamedi et al. 2008). The single class IV NFR is located telomere-proximally to $t$ lh2, and its elimination requires the activities of the SHREC components Chp2, Clr3, and Mit1 (Fig. 6). The elimination of class V NFRs requires Clr4, but no single component tested recapitulates the phenotype of $c \operatorname{lr} 4 \Delta$ here, indicating either redundancy between the factors tested or a novel mechanism of recognition of Clr4-dependent methylation. Although further work will be needed to understand these distinct requirements, these results indicate that the core silencing machinery consists of a molecular toolbox that is differentially deployed in a manner that depends on the task required of it at a particular sequence. Our analysis of chromatin structure may help to explain previous observations that show that loss of the HP1 proteins can result in quantitative differences in the levels of RNA accumulation, depending on the region/reporter gene location assayed (Motamedi et al. 2008; Sadaie et al. 2008).

\section{Mechanism of NFR elimination: Clr3 HDAC activity antagonizes RSC}

SHREC is related to the mammalian NuRD complex, and our studies dissected the relative roles of its two catalytic activities in controlling nucleosome positions. We found that the HDAC activity of the Clr3 subunit of the SHREC effector complex is important for NFR elimination at all sites affected by Clr4 except one, whereas, surprisingly, the Mit1 ATPase acts at considerably fewer sites. We tested the hypothesis that the HDAC activity of Clr3 promotes NFR elimination by inhibiting the recruitment and/or activity of a factor required for NFR formation. A candidate for such a factor is the conserved RSC remodeling complex, which promotes NFR formation in $S$. cerevisiae (Hartley and Madhani 2009) and is conserved in S. pombe (Monahan et al. 2008). Multiple subunits of RSC contain bromodomains and acetyl-lysine-binding domains, and RSC recruitment is promoted by histone tail acetylation (Carey et al. 2006). Indeed, we found that the requirement for Clr3 deacetylase activity in NFR elimination could be strongly or partially bypassed by crippling the $S$. pombe RSC chromatin remodeling complex by deletion of the gene encoding the nonessential Rsc1 subunit. Class I NFRs displayed the strongest suppression phenotype. Correspondingly, we observed that Clr3 activity antagonized the recruitment of RSC at these NFRs. At NFRs where partial suppression was observed, we did not observe a change in RSC recruitment, suggesting that either our assay sensitivity was insufficient to detect a difference in recruitment, or RSC activity rather than recruitment is affected by acetylation at these sites. Raising the possibility that RSC activity could be regulated by a deacetylase, a subunit of RSC itself, Rsc4, has been shown to be acetylated in S. cerevisiae (VanDemark et al. 2007).

\section{Silencing-resistant NFRs at heterochromatin boundaries}

Our data illuminate the chromatin structure at boundary elements in S. pombe that limit the spread of heterochromatin by not well-understood mechanisms. As mentioned above, those that flank the silent mat2/3 require clusters of binding sites for the PolIII transcription factor TFIIIC. Our data show that these coincide with NFRs that are unaffected by heterochromatin. We observed 
similar results at tRNA genes in the imr region of the centromere that, being PolIII transcripts, also have binding sites for TFIIIC. These observations suggest that gaps in the nucleosome array are not only a substrate for heterochromatin action, but also, when associated with TFIIIC sites, may regulate its lateral spread by being resistant to the NFR-shrinking activities of the silencing machinery. In this regard, it is interesting to note that, of all sites in the genome, RSC in S. cerevisiae displays the highest occupancy at tRNA genes (Ng et al. 2002); thus, it may be that the ability of boundary elements to recruit RSC strongly promotes their resistance to NFR elimination.

\section{Concluding remarks}

This work defines chromatin structural changes induced by the machinery required for heterochromatic silencing in $S$. pombe. We found that the nucleosome spacing of $S$. pombe heterochromatin was less periodic than that of euchromatin. We observed that silencing factors induce the elimination of NFRs, including those not associated with transcriptional initiation sites. Significantly, different combinations of silencing factors were required at different DNA sites, which may explain the need for complexity in the silencing machinery. This study suggests that NFR elimination by the combinatorial action of silencing factors is a key component to the mechanism of heterochromatin silencing.

\section{Materials and methods}

S. pombe strains

Strains described in this study are listed in Supplemental Table S1.

Probe generation for nucleosome positioning experiments

Mononucleosomes were prepared from cells that were grown to mid-log phase $\left(\mathrm{OD}_{600} \sim 0.7-0.8\right)$, cross-linked, and spheroplasted. Mononucleosomes were obtained from the MNase-treated spheroplasts as detailed in the Supplemental Material.

\section{Tiling array design}

The $4 \mathrm{x} 44 \mathrm{k}$ tiling array used throughout most of this study tiled the following loci at 4-bp resolution: cen1, the mating type locus (MTL), tel2R, mei4-act1, his3, ade6, and nmt1-gut2. It should be noted that the following elements on the array have high sequence similarity to other regions of the genome, leading to a high enrichment of nonunique probes: cen $1, t e l 2 R$, and $I R$ repeats within the $M T L$; the mat3-M element in the $M T L$; and the cenH element in the MTL. For these regions, the nucleosome occupancy should be considered as an average of the nucleosome occupancy for this loci and other similar loci. The second tiling array was used mainly to generate the data in Figure 1, and Supplemental Figures S3, C and D, and S7 tiled the following loci: sod1-nup124, mek1, and mei4-act1. See Supplemental Figure $\mathrm{S} 1 \mathrm{~B}$ regarding the uniqueness of probes.

Microarray hybridization and data processing of nuclesome occupancy data

Probes were generated from mononucleosomal DNA, and were hybridized against probes generated from genomic DNA lightly digested with MNase onto the microarrays as described in the Supplemental Material.

Raw data were processed as described (Hartley and Madhani 2009). Briefly, a mononucleosome:genomic ratio was calculated by taking the $\log _{2}$ ratio of each probe after subtracting the median background intensity from the foreground intensity. The probe ratios were then normalized using a LOESS regression algorithm with a smooth value of 0.4. The nucleosome enrichment plots represent the mean of two biological replicates that has been smoothed using a centered moving average of 19 probes (76 bp, or half the size of mononucleosomal-sized DNA).

Microarray data can be accessed at the NCBI Gene Expression Omnibus under the accession number GSE19596.

\section{Significance test}

To analyze significant changes within a data set, we created a difference map where a wild-type data set was subtracted from the mutant data set. The data sets that were used represented the moving average of the normalized mean $\log _{2}$ replicate data over 19 probes. We calculated a $Z$-score $(z)$ that corresponded to one event occurring by chance in $n$, the number of data points within the difference map, by using the following function in the analysis software: R: $z=$ qnorm $(1 / n)$. From that $z$, the standard deviation $(\sigma)$ and the mean $(\mu)$ of the difference map was used to calculate $x$, the cutoff, using the the following equation: $x=\left[z^{\star} \sigma\right]-\mu$. Any of the 15 regions identified in Figure 2 with at least 25 continuous probes (100 bp) above this cutoff was considered to display a significant change.

Additional methods are available in the Supplemental Material.

\section{Acknowledgments}

We thank Geeta Narlikar and Stavros Lomvardas for critical reading of this paper. This work was supported by a grant to H.D.M. from the National Institutes of Health (GM071801). H.D.M. is a fellow of the Leukemia and Lymphoma Society. J.F.G. was supported by an NIH/NIGMS IMSD predoctoral fellowship. P.D.H. was supported by an NIH Kirschstein predoctoral fellowship. P.A.D. was supported by the University of California at San Francisco Medical Scientist Training Program. J.G. and H.D.M. designed the study. J.G. and P.A.D. constructed the strains used. J.G. and P.A.D. performed the experiments. P.D.H. wrote the software to process the microarray data. H.E-S. performed the Fourier analysis. J.G. and H.D.M wrote the manuscript. All authors contributed to editing the manuscript.

\section{Note added in proof}

After this work was accepted for publication, we learned that Shiv Grewal's laboratory (National Cancer Institute) also found a role for the HDAC activity of Clr3 in promoting NFR elimination within heterochromatic domains, and also identified heterochromatin-resistant nucleosome-free regions at heterochromatic boundaries (S Grewal, pers. comm.).

\section{References}

Allshire R, Javerzat J, Redhead NJ, Cranston G. 1994. Position effect variegation at fission yeast centromeres. Cell 76: 157-169.

Ayoub N, Goldshmidt I, Lyakhovetsky R, Cohen A. 2000. A fission yeast repression element cooperates with centromere-like sequences and defines a mat silent domain boundary. Genetics 156: 983-994. 
Brownell JE, Zhou J, Ranalli T, Kobayashi R, Edmondson DG, Roth SY, Allis CD. 1996. Tetrahymena histone acetyltransferase A: A homolog to yeast Gcn5p linking histone acetylation to gene activation. Cell 84: 843-851.

Bühler M, Moazed D. 2007. Transcription and RNAi in heterochromatic gene silencing. Nat Struct Mol Biol 14: 1041-1048.

Carey M, Li B, Workman JL. 2006. RSC exploits histone acetylation to abrogate the nucleosomal block to RNA polymerase II elongation. Mol Cell 24: 481-487.

Chen L, Widom J. 2005. Mechanism of transcriptional silencing in yeast. Cell 120: 37-48.

Chen C, Yang TP. 2001. Nucleosomes are translationally positioned on the active allele and rotationally positioned on the inactive allele of the HPRT promoter. Mol Cell Biol 21: 7682-7695.

Chen ES, Zhang K, Nicolas E, Cam HP, Zofall M, Grewal SIS. 2008. Cell cycle control of centromeric repeat transcription and heterochromatin assembly. Nature 451: 734-737.

Dillon N. 2004. Heterochromatin structure and function. Biol Cell 96: 631-637.

Djupedal I, Ekwall K. 2009. Epigenetics: Heterochromatin meets RNAi. Cell Res 19: 282-295.

Ekwall K, Nielsen O, Ruusala T. 1991. Repression of a mating type cassette in the fission yeast by four DNA elements. Yeast 7: 745-755.

Fischer T, Cui B, Dhakshnamoorthy J, Zhou M, Rubin C, Zofall M, Veenstra TD, Grewal SIS. 2009. Diverse roles of HP1 proteins in heterochromatin assembly and functions in fission yeast. Proc Natl Acad Sci 106: 8998-9003.

Fyodorov DV, Blower MD, Karpen GH, Kadonaga JT. 2004. Acf1 confers unique activities to ACF/CHRAC and promotes the formation rather than disruption of chromatin in vivo. Genes Dev 18: 170-183.

Gartenberg M. 2009. Heterochromatin and the cohesion of sister chromatids. Chromosome Res 17: 229-238.

Grewal S, Elgin SC. 2007. Transcription and RNA interference in the formation of heterochromatin. Nature 447: 399406.

Grewal SIS, Jia S. 2007. Heterochromatin revisited. Nat Rev Genet 8: 35-46.

Hansen KR, Ibarra PT, Thon G. 2006. Evolutionary-conserved telomere-linked helicase genes of fission yeast are repressed by silencing factors, RNAi components and the telomerebinding protein Tazl. Nucleic Acids Res 34: 78-88.

Hartley PD, Madhani HD. 2009. Mechanisms that specify promoter nucleosome location and identity. Cell 137: 445-458.

Hassan AH, Awad S, Al-Natour Z, Othman S, Mustafa F, Rizvi TA. 2007. Selective recognition of acetylated histones by bromodomains in transcriptional co-activators. Biochem $J$ 402: 125-133.

Heitz E. 1928. Das heterochromatin der moose. Jahrb Wiss Bot 69: $762-818$.

Jae Yoo E, Kyu Jang Y, Ae Lee M, Bjerling P, Bum Kim J, Ekwall K, Hyun Seong R, Dai Park S. 2002. Hrp3, a chromodomain helicase/ATPase DNA binding protein, is required for heterochromatin silencing in fission yeast. Biochem Biophys Res Commun 295: 970-974.

Jia S, Noma K-I, Grewal SIS. 2004a. RNAi-independent heterochromatin nucleation by the stress-activated ATF/CREB family proteins. Science 304: 1971-1976.

Jia S, Yamada T, Grewal S. 2004b. Heterochromatin regulates cell type-specific long-range chromatin interactions essential for directed recombination. Cell 119: 469-480.

Jin Q-W, Pidoux AL, Decker C, Allshire RC, Fleig U. 2002. The mal2p protein is an essential component of the fission yeast centromere. Mol Cell Biol 22: 7168-7183.
Kasten M, Szerlong H, Erdjument-Bromage H, Tempst P, Werner M, Cairns BR. 2004. Tandem bromodomains in the chromatin remodeler RSC recognize acetylated histone H3 Lys14. $E M B O$ J 23: $1348-1359$.

Kayne PS, Kim UJ, Han M, Mullen JR, Yoshizaki F, Grunstein M. 1988. Extremely conserved histone $\mathrm{H} 4 \mathrm{~N}$ terminus is dispensable for growth but essential for repressing the silent mating loci in yeast. Cell 55: 27-39.

Lantermann A, Strålfors A, Fagerström-Billai F, Korber P, Ekwall K. 2009. Genome-wide mapping of nucleosome positions in Schizosaccharomyces pombe. Methods 48: 218-225.

Lantermann AB, Straub T, Strålfors A, Yuan G-C, Ekwall K, Korber P. 2010. Schizosaccharomyces pombe genome-wide nucleosome mapping reveals positioning mechanisms distinct from those of Saccharomyces cerevisiae. Nat Struct Mol Biol 17: 251-257.

Lejeune E, Bortfeld M, White SA, Pidoux AL, Ekwall K, Allshire RC, Ladurner AG. 2007. The chromatin-remodeling factor FACT contributes to centromeric heterochromatin independently of RNAi. Curr Biol 17: 1219-1224.

Lomberk G, Wallrath L, Urrutia R. 2006. The Heterochromatin Protein 1 family. Genome Biol 7: 228. doi: 10.1186/gb-20067-7-228.

Monahan BJ, Villén J, Marguerat S, Bähler J, Gygi SP, Winston F. 2008. Fission yeast SWI/SNF and RSC complexes show compositional and functional differences from budding yeast. Nat Struct Mol Biol 15: 873-880.

Motamedi MR, Hong E-JE, Li X, Gerber S, Denison C, Gygi S, Moazed D. 2008. HP1 proteins form distinct complexes and mediate heterochromatic gene silencing by nonoverlapping mechanisms. Mol Cell 32: 778-790.

Murakami H, Goto D, Toda T, Chen E, Grewal S, Martienssen R, Yanagida M. 2007. Ribonuclease activity of Dis3 is required for mitotic progression and provides a possible link between heterochromatin and kinetochore function. PLOS One 2: e317. doi: 10.1371/journal.pone.0000317.

$\mathrm{Ng} \mathrm{HH}$, Robert F, Young RA, Struhl K. 2002. Genome-wide location and regulated recruitment of the RSC nucleosomeremodeling complex. Genes Dev 16: 806-819.

Noma K, Cam H, Maraia RJ, Grewal S. 2006. A role for TFIIIC transcription factor complex in genome organization. Cell 125: 859-872.

Peng JC, Karpen GH. 2008. Epigenetic regulation of heterochromatic DNA stability. Curr Opin Genet Dev 18: 204-211.

Routh A, Sandin S, Rhodes D. 2008. Nucleosome repeat length and linker histone stoichiometry determine chromatin fiber structure. Proc Natl Acad Sci 105: 8872-8877.

Sadaie M, Kawaguchi R, Ohtani Y, Arisaka F, Tanaka K, Shirahige K, Nakayama J-I. 2008. Balance between distinct HP1 family proteins controls heterochromatin assembly in fission yeast. Mol Cell Biol 28: 6973-6988.

Scott KC, Merrett SL, Willard HF. 2006. A heterochromatin barrier partitions the fission yeast centromere into discrete chromatin domains. Curr Biol 16: 119-129.

Segal E, Widom J. 2009a. From DNA sequence to transcriptional behaviour: A quantitative approach. Nat Rev Genet 10: 443456.

Segal E, Widom J. 2009b. What controls nucleosome positions? Trends Genet 25: 335-343.

Sekinger EA, Gross DS. 2001. Silenced chromatin is permissive to activator binding and PIC recruitment. Cell 105: 403414.

Smirnova JB, McFarlane RJ. 2002. The unique centromeric chromatin structure of Schizosaccharomyces pombe is maintained during meiosis. I Biol Chem 277: 1981719822. 
Song JS, Liu X, Liu XS, He X. 2008. A high-resolution map of nucleosome positioning on a fission yeast centromere. Genome Res 18: 1064-1072.

Sugiyama T, Cam H, Sugiyama R, Noma K, Zofall M, Kobayashi R, Grewal S. 2007. SHREC, an effector complex for heterochromatic transcriptional silencing. Cell 128: 491-504.

Sun FL, Cuaycong MH, Elgin SC. 2001. Long-range nucleosome ordering is associated with gene silencing in Drosophila melanogaster pericentric heterochromatin. Mol Cell Biol 21: 2867-2879.

Takahashi K, Chen ES, Yanagida M. 2000. Requirement of Mis6 centromere connector for localizing a CENP-A-like protein in fission yeast. Science 288: 2215-2219.

Thon G, Verhein-Hansen J. 2000. Four chromo-domain proteins of Schizosaccharomyces pombe differentially repress transcription at various chromosomal locations. Genetics 155: 551-568.

Thon G, Bjerling KP, Nielsen IS. 1999. Localization and properties of a silencing element near the mat3-M mating-type cassette of Schizosaccharomyces pombe. Genetics 151: 945-963.

VanDemark AP, Kasten MM, Ferris E, Heroux A, Hill CP, Cairns BR. 2007. Autoregulation of the rsc 4 tandem bromodomain by gen 5 acetylation. Mol Cell 27: 817-828.

Vega-Palas MA, Venditti S, Di Mauro E. 1998. Heterochromatin organization of a natural yeast telomere. Changes of nucleosome distribution driven by the absence of Sir3p. I Biol Chem 273: 9388-9392.

Verdel A, Jia S, Gerber S, Sugiyama T, Gygi S, Grewal S, Moazed D. 2004. RNAi-mediated targeting of heterochromatin by the RITS complex. Science 303: 672-676.

Verschure PJ, van der Kraan I, de Leeuw W, van der Vlag J, Carpenter AE, Belmont AS, van Driel R. 2005. In vivo HP1 targeting causes large-scale chromatin condensation and enhanced histone lysine methylation. Mol Cell Biol 25: 4552-4564.

Volpe T, Schramke V, Hamilton GL, White SA, Teng G, Martienssen RA, Allshire RC. 2003. RNA interference is required for normal centromere function in fission yeast. Chromosome Res 11: 137-146.

Walfridsson J, Bjerling P, Thalen M, Yoo E-J, Park SD, Ekwall K. 2005. The CHD remodeling factor Hrp1 stimulates CENP-A loading to centromeres. Nucleic Acids Res 33: 2868-2879.

Wallrath LL, Elgin SC. 1995. Position effect variegation in Drosophila is associated with an altered chromatin structure. Genes Dev 9: 1263-1277.

White SA, Allshire RC. 2008. RNAi-mediated chromatin silencing in fission yeast. Curr Top Microbiol Immunol 320: 157-183.

Wilhelm BT, Marguerat S, Watt S, Schubert F, Wood V, Goodhead I, Penkett CJ, Rogers J, Bähler J. 2008. Dynamic repertoire of a eukaryotic transcriptome surveyed at single-nucleotide resolution. Nature 453: 1239-1243.

Wu C, Wong YC, Elgin SC. 1979. The chromatin structure of specific genes: II. Disruption of chromatin structure during gene activity. Cell 16: 807-814.

Yuan G-C, Liu Y-J, Dion MF, Slack MD, Wu LF, Altschuler SI, Rando OJ. 2005. Genome-scale identification of nucleosome positions in S. cerevisiae. Science 309: 626-630. 


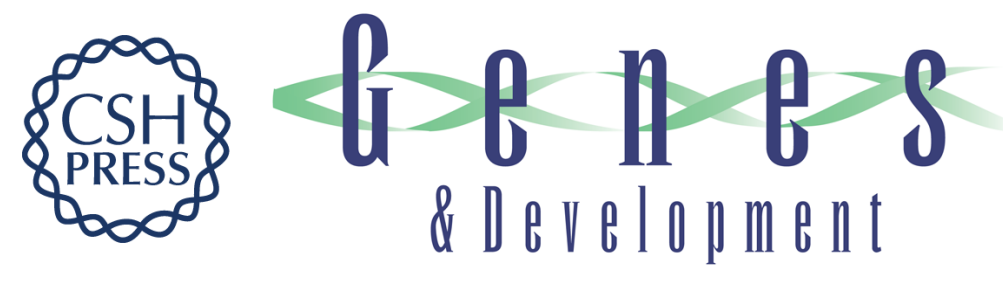

\section{Combinatorial, site-specific requirement for heterochromatic silencing factors in the elimination of nucleosome-free regions}

Jennifer F. Garcia, Phillip A. Dumesic, Paul D. Hartley, et al.

Genes Dev. 2010, 24: originally published online July 30, 2010

Access the most recent version at doi:10.1101/gad.1946410

\section{Supplemental http://genesdev.cshlp.org/content/suppl/2010/07/21/gad.1946410.DC1 \\ Material}

References This article cites 60 articles, 19 of which can be accessed free at:

http://genesdev.cshlp.org/content/24/16/1758.full.html\#ref-list-1

License

Email Alerting

Receive free email alerts when new articles cite this article - sign up in the box at the top

Service

right corner of the article or click here.

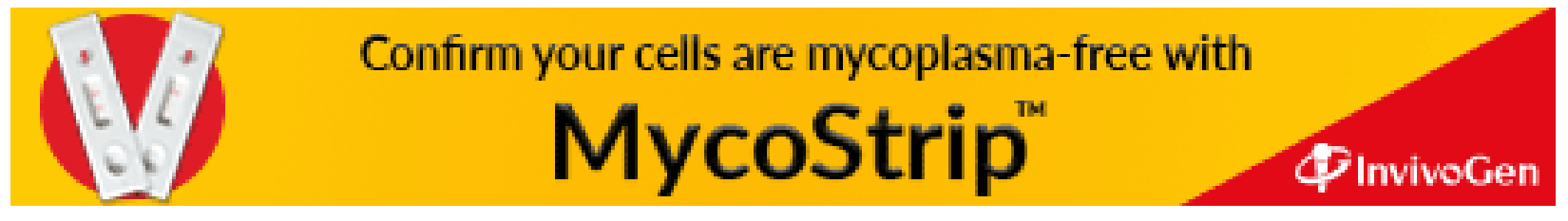

